\title{
Keimel's problem on the algebraic axiomatization of convexity
}

\author{
A. Komorowski, A. B. Romanowska and J. D. H. Smith
}

\begin{abstract}
Convex sets may be viewed as algebras equipped with a set of binary convex combinations that is indexed by the open unit interval of real numbers. Convex sets generate the variety of barycentric algebras, which also includes semilattices where the semilattice multiplication is repeated uncountably many times. Barycentric algebras are defined by three axioms: idempotence, skew-commutativity, and skew-associativity. Since the skew-associativity axiom is rather complicated, Klaus Keimel has asked whether it can simply be replaced by the entropic law. It turns out that the answer is negative. The counterexamples presented and studied in this paper are known as threshold barycentric algebras, depending on a threshold taken from the left-hand side of the closed unit interval. They offer an entire spectrum of algebras, ranging from barycentric algebras for threshold 0 to commutative idempotent entropic groupoids for threshold $1 / 2$.
\end{abstract}

Mathematics Subject Classification. 08A99, 52A01.

Keywords. Entropic algebra, Barycentric algebra, Convex set, Semilattice, Self-distributive, Convexity.

\section{Introduction}

Real convex sets may be presented algebraically as sets with binary operations given by weighted means, the weights being taken from the open unit interval $\left.I^{\circ}=\right] 0,1[$ in the set of reals. The class of convex sets is a quasivariety (defined by certain implications), and generates the variety (defined by identities) of socalled barycentric algebras (Section 2). Both these classes have a well-developed theory, a special case of the general theory of modes (idempotent and entropic

Presented by K. A. Kearnes.

The first author's research was supported by the Warsaw University of Technology under grant number 504/03340/1120. 
algebras, Section 3) $[3,4,11,12,13,14,15,16,18]$. In particular, like all modes, barycentric algebras implement self-distributivity in the sense of [2].

The variety of barycentric algebras contains convex sets, semilattices (where all the barycentric operations coincide), and certain semilattice sums of convex sets. It is defined by three types of axioms: idempotence, skewcommutativity, and skew-associativity, the latter two implying the entropic property that each operation is a homomorphism. Since the skew-associativity axiom is rather complicated, Klaus Keimel asked whether it may simply be replaced by entropicity alone [8]. In this paper, we show that the answer is negative. We construct an entropic algebra, of the same type as barycentric algebras, which is idempotent and skew-commutative, but is not skew-associative (Theorem 5.1).

Together with barycentric algebras, the counterexample belongs to a much larger family of algebras described as threshold barycentric algebras, which model the concept of threshold convexity introduced in Section 4. Take a fixed element $t$ of $[0,1 / 2]$ known as the threshold. Then the full open real interval $I^{\circ}$ of binary barycentric operations is replaced by the barycentric operations indexed by the subinterval $[t, 1-t] \cap I^{\circ}$ of $I^{\circ}$, together with so-called extreme operations: left-zero operations (left projections) indexed by elements of the interval ] $0, t$ [, and right-zero operations (right projections) indexed by elements of the interval ] $1-t, 1[$. These extreme operations implement projection dimonoids in the sense of [7, Ex. 1.3(b)], [10, Ex. 3.2], [20, Ex. 2.2].

Varieties of threshold barycentric algebras fall into three classes:

- The variety $\mathcal{B}^{0}$ of threshold-0 barycentric algebras coincides with the variety $\mathcal{B}$ of usual (skew-associative) barycentric algebras.

- For $0<t<1 / 2$, threshold- $t$ barycentric algebras are not skew-associative (Theorem 6.4). But the variety $\mathcal{B}^{t}$ of threshold- $t$ barycentric algebras is equivalent to the variety $\overline{\mathcal{B}}$ of extended barycentric algebras (Theorem 9.4). Extended barycentric algebras are defined like usual barycentric algebras, but with the closed unit interval $I$ of barycentric operations replacing the open interval $I^{\circ}$, and thus including projection dimonoid structure.

- The variety $\mathcal{B}^{1 / 2}$ of threshold-(1/2) barycentric algebras is equivalent to the variety $\overline{\mathcal{C B M}}$ of extended commutative binary modes (Theorem 7.2). These algebras have one binary commutative mode operation that is given by $t=1 / 2$, together with projection dimonoid structures.

Thus threshold barycentric algebras offer an entire spectrum of algebras, ranging from the usual barycentric algebras at one end to the commutative binary modes at the other. In [17], it was shown that barycentric algebras may be used to replace Boolean networks in systems biology with models offering a more realistic tracking of the biochemistry. Because the Boolean networks correspond to the use of threshold-(1/2) convexity in that application, the threshold $t$ may be viewed as a parameter providing a smooth transition between the two classes of models. 
Theorem 8.5 shows that for a threshold $0<t<1 / 2$, finite-dimensional simplices (free barycentric algebras) are also generated by their vertices under the basic threshold- $t$ barycentric operations. The golden section makes an appearance in the analysis underlying the proof of the theorem (Remark 8.3).

Section 10 discusses the varieties $\mathcal{S}^{t}$ of threshold- $t$ semilattices (Proposition 10.3). Extended semilattices are defined by adding projection operations to semilattices, in similar fashion to extended barycentric algebras and extended commutative binary modes. Then for each positive threshold $t$, the variety $\mathcal{S}^{t}$ of threshold- $t$ semilattices is equivalent to the variety of extended semilattices (Proposition 10.6).

Sections 11 and 12 investigate the behavior of threshold- $t$ barycentric varieties within the full variety $\mathcal{S C}$ of skew-commutative modes of barycentric algebra type $I^{\circ} \times\{2\}$. Theorem 12.3 identifies the meet $\mathcal{B}^{s} \wedge \mathcal{B}^{t}$ for distinct thresholds $s$ and $t$, while Theorem 12.6 identifies the join $\mathcal{B}^{s} \vee \mathcal{B}^{t}$.

Background facts concerning barycentric algebras and commutative binary modes are summarized in Sections 2 and 3. For more information on such algebras, and modes in general, we refer the reader to the monographs $[14,16]$. We use notation and conventions similar to those of the cited monographs and [21].

\section{Barycentric algebras}

Let $F$ be a field. A unary operation of complementation is defined by

$$
p^{\prime}=1-p
$$

for $p \in F$. A binary dual multiplication is defined by

$$
p \circ q=\left(p^{\prime} q^{\prime}\right)^{\prime}
$$

for $p, q \in F$. A binary operation of implication is defined by

$$
p \rightarrow q= \begin{cases}1 & \text { if } p=0 \\ q / p & \text { otherwise }\end{cases}
$$

for $p, q \in F$. Note that for $F=\mathrm{GF}(2)$, the definition (2.1) recovers the usual Boolean implication.

Let $I^{\circ}$ denote the open unit interval

$$
] 0,1[=\{p \in \mathbb{R} \mid 0<p<1\} .
$$

Then an algebra $\left(A, \underline{I}^{\circ}\right)$, with a binary operation $p$ for each operator $p \in I^{\circ}$, is said to be a barycentric algebra if it satisfies the identities

$$
x x \underline{p}=x
$$

of idempotence,

$$
x y \underline{p}=y x \underline{p^{\prime}}
$$

of skew-commutativity, and

$$
[x y \underline{p}] z \underline{q}=x[y z \underline{(p \circ q \rightarrow q)}] \underline{p \circ q}
$$


of skew-associativity for all $p, q \in I^{\circ}$. The class $\mathcal{B}$ of barycentric algebras forms a variety $[3,4,11,12,13,14,15,16,18]$.

Convex subsets of real affine spaces are barycentric algebras $\left(C, \underline{I}^{\circ}\right)$ under the operations

$$
x y \underline{p}=x p^{\prime}+y p=x(1-p)+y p
$$

for each $p \in I^{\circ}$. They form the subquasivariety $\mathcal{C}$ of the variety $\mathcal{B}$ defined by the cancellation laws

$$
(x y \underline{p}=x z \underline{p}) \rightarrow(y=z)
$$

for all operations $p$ of $\underline{I}^{\circ}$. In particular, cancellative members of $\mathcal{B}$ are precisely convex sets (see $[\overline{16}$, Th. 5.8 .6$]$ ), and the class $\mathcal{C}$ generates the variety $\mathcal{B}$. Other examples are provided by semilattices, idempotent, commutative semigroups $(S, \cdot)$, considered as barycentric algebras with the "stammered" operation $x$. $y=x y p$ for all $p \in I^{\circ}$. Note that semilattice barycentric algebras $\left(S, \underline{I}^{\circ}\right)$ satisfy the identities $x y p=x y \underline{r}$ for all $p, r \in I^{\circ}$. They are also interpreted as ordered sets, meet semilattices with the ordering relation defined by $x \leq y$ if and only if $x \cdot y=x$. The variety $\mathcal{S}$ of semilattice barycentric algebras is the only non-trivial proper subvariety of the variety $\mathcal{B}$.

The structure of general barycentric algebras $\left(A, \underline{I}^{\circ}\right)$ is described by the following theorem.

Theorem 2.1 ([16, $§ 7.5])$.

(a) Each barycentric algebra $\left(A, \underline{I}^{\circ}\right)$ has a homomorphism onto its semilattice replica, with open convex sets as the corresponding fibres.

(b) Each barycentric algebra $\left(A, \underline{I}^{\circ}\right)$ is a subalgebra of a Ptonka sum of convex sets over its semilattice replica.

Barycentric algebras may also be defined as algebras $(A, \underline{I})$, where $I=$ $[0,1]$ is the closed unit interval of $\mathbb{R}$, with the operations $\underline{0}$ and $\underline{1}$ defined by

$$
x y \underline{0}=x \text { and } x y \underline{1}=y
$$

$[3,4,12,13,18]$. The class $\overline{\mathcal{B}}$ of barycentric algebras defined in this way is also a variety. It is specified by the identities defining $\mathcal{B}$ together with the additional identities (2.6). Examples are provided by convex sets and semilattices considered as usual barycentric algebras, with two additional operations $\underline{0}$ and $\underline{1}$. We will refer to members of $\overline{\mathcal{B}}$ as extended barycentric algebras.

\section{Entropic algebras and modes}

For an algebra $(A, \Omega)$ equipped with a set $\Omega$ of binary operations, and $\omega, \varphi \in \Omega$, consider the entropic identity

$$
(x y \omega)(z t \omega) \varphi=(x z \varphi)(y t \varphi) \omega .
$$

Algebras satisfying the identities (3.1) for all pairs of (equal or unequal) operations from $\Omega$ are called entropic algebras. Idempotent entropic algebras are called modes. Left-zero bands (defined by the left projection $x \triangleleft y=x$ ) and right-zero bands (defined by the right projection $x \triangleright y=y$ ), as well as semilattices and more general semigroup modes (namely normal bands), all form 
modes. Note that the operations $\underline{0}$ and $\underline{1}$, as defined in (2.6), are respectively left-zero and right-zero operations.

Further examples of modes are provided by barycentric and extended barycentric algebras, and by affine spaces and their reducts. (Here, affine spaces over a field $F$ of odd characteristic are defined as algebras $(A, \underline{F})$ with binary operations $p$ given by (2.4) for each $p \in F$. In a similar way, one may define affine spaces over commutative rings of odd characteristic [16]). Among groupoids (or magmas), algebras with one binary operation, we will be especially interested in commutative binary modes, groupoid modes $(A, \star)$ with a commutative multiplication $\star$, and in extended commutative binary modes $(A, \star, \triangleleft, \triangleright)$, equipped with additional left and right projections.

Commutative binary modes form a variety, written as $\mathcal{C B M}$ [16, Ch. 5]. The cancellative members of this variety are known to be subreducts of quasigroup modes, or equivalently $1 / 2$-subreducts of affine spaces over the ring of rational dyadic numbers, $\left.\mathbb{D}=\overline{\left\{2^{n}\right.} m \mid m, n \in \mathbb{Z}\right\}[5$, Th. 5.3.1], [16, Chs. 6, 7]. The unit interval $\mathbb{D}_{1}=\mathbb{D} \cap[0,1]$ of $\mathbb{D}$, considered as a groupoid under the operation $\star$ of arithmetical mean, generates the variety $\mathcal{C} \mathcal{B} \mathcal{M}$. The algebra $\left(\mathbb{D}_{1}, \star\right)$ is the free commutative binary mode on two free generators 0 and 1 [16, Ch. 5]. Now $\left(\mathbb{D}_{1}, \star\right)$ and $\left(\mathbb{D}_{1}, \underline{\mathbb{D}}_{1}^{\circ}\right)$, where $\underline{\mathbb{D}}_{1}^{\circ}$ is the set of operations $\underline{d}$ for $d \in \mathbb{D}_{1}^{\circ}=\mathbb{D}_{1} \backslash\{0,1\}$, are (clonally or term) equivalent [16, $\left.\S 1.4\right]$. More generally, subalgebras of convex sets considered as algebras with $\star$ as the basic operation, or with the operations of $\underline{\mathbb{D}}_{1}^{\circ}$ as the basic operations, are equivalent.

The structure of commutative binary modes is generally similar to the structure of barycentric algebras as summarized in Theorem 2.1.

Theorem 3.1 ([16, $§ 7.5])$.

(a) Each commutative binary mode $(A, \star)$ has a homomorphism onto its semilattice replica, with cancellative subgroupoids as the corresponding fibres.

(b) Each commutative binary mode $(A, \star)$ is a subalgebra of a Ptonka sum of cancellative commutative binary modes over its semilattice replica.

\section{Threshold convexity}

Convexity is described algebraically using the binary operations (2.4) of weighted means, for complementary weights $p^{\prime}, p$ taken from the open unit interval. The new concept of threshold convexity replaces weighted means whose weights differ widely by projections to the most heavily weighted argument.

Definition 4.1. Consider a real number $0 \leq t \leq 1 / 2$, known as the threshold.

(a) For elements $x, y$ of a convex set $C$, define

$$
x y \underline{\underline{r}}= \begin{cases}x & \text { if } r<t \\ x y \underline{r}=x(1-r)+y r & \text { if } t \leq r \leq 1-t \\ y & \text { if } r>1-t\end{cases}
$$


for $0<r<1$. Then the binary operations $\underline{r}$ are described as thresholdconvex combinations.

(b) A threshold-convex combination $\underline{\underline{r}}$ is respectively known as small, moderate, or large when $r$ lies in the respective members $] 0, t[,[t, 1-t]] 1-t,, 1[$ of the partition \{] $0, t[,[t, 1-t]] 1-t,, 1[\}$ of the open unit interval $] 0,1[$. Together, small and large threshold-convex combinations are described as extreme.

Remark 4.2. The threshold-convex combinations specialize to the standard convex combinations when the threshold is set to zero, so that there are no extreme convex combinations in that case.

Lemma 4.3. Set a threshold $0 \leq t \leq 1 / 2$. Let $C$ be a convex set. Then under the threshold-convex combinations $\underline{\underline{r}}$ for $0<r<1$, the algebra $\left(C, \underline{\underline{I}}^{o}\right)$ forms an idempotent algebra, satisfying the skew-commutativity identity (2.2) written as:

$$
x y \underline{\underline{r}}=y x \underline{r^{\prime}} .
$$

Proposition 4.4. Set a threshold $0 \leq t \leq 1 / 2$. Let $C$ be a convex set. Then under the threshold-convex combinations $\underline{\underline{r}}$ for $0<r<1$, the algebra $\left(C, \underline{\underline{I}}^{o}\right)$ forms an entropic algebra.

Proof. For elements $u, v, w, x$ of $C$ and $0<r, s<1$, the entropic identity

$$
(u v \underline{\underline{r}})(w x \underline{\underline{r}}) \underline{\underline{s}}=(u w \underline{\underline{s}})(v x \underline{\underline{s}}) \underline{\underline{r}}
$$

must be verified. Note that the identity is symmetrical in the operations $\underline{\underline{r}}$ and $\underline{s}$.

There are various cases for the evaluation of the two sides of (4.3), according to whether the threshold-convex combinations $\underline{\underline{r}}$ and $\underline{\underline{s}}$ are small, moderate, or large. If both are small, the algebra $(C, \underline{\underline{r}}, \underline{\underline{s}})$ is a left zero semigroup (taken with repeated operations). As such, it is entropic. Dually, $(C, \underline{\underline{r}}, \underline{\underline{s}})$ is entropic as a right zero semigroup if both $\underline{\underline{r}}$ and $\underline{\underline{s}}$ are large. Again, $(C, \underline{\underline{\underline{r}}}, \underline{\underline{\underline{s}}})$ is entropic as a reduct of a barycentric algebra if both $\underline{\underline{r}}$ and $\underline{\underline{s}}$ are moderate.

If one combination (say $\underline{\underline{r}}$ ) is small, and the other is moderate, then both sides of (4.3) reduce to $u \bar{w} \underline{\underline{s}}$. The case where one is large, and the other moderate, is dual. Finally, suppose $\underline{\underline{r}}$ is small and $\underline{\underline{s}}$ is large. Then the lefthand side of (4.3) evaluates to $u w \underline{\underline{s}}=w$, while the right-hand side evaluates to $w x \underline{\underline{r}}=w$.

Corollary 4.5. Threshold-convex sets $\left(C, \underline{\underline{I}}^{o}\right)$ are modes.

\section{Keimel's problem}

Theorem 5.1. In the specification of barycentric algebras, the axiom of skewassociativity cannot be replaced by the axiom of entropicity.

Proof. Consider the closed unit interval $I=[0,1]$ as a convex set. Set a threshold of $t=1 / 2$, and take $I$ as an algebra under all the threshold-convex combinations, as outlined in Section 4. According to Lemma 4.3, I is idempotent 
and skew-commutative. By Proposition 4.4, I is entropic. But it will now be shown that $I$ is not skew-associative: the identity (2.3) written as

$$
[x y \underline{\underline{p}}] z \underline{\underline{q}}=x[y z \underline{\underline{(p \circ q \rightarrow q)}}] \underline{\underline{p \circ q}}
$$

is violated.

In the identity (5.1), take $p=q=1 / 2$. Thus $p \circ q=\left(p^{\prime} q^{\prime}\right)^{\prime}=(1 / 4)^{\prime}=3 / 4$, and $p \circ q \rightarrow q=3 / 4 \rightarrow 1 / 2=(1 / 2) /(3 / 4)=2 / 3$. Now take $x=y=0$ and $z=1$. Then the left-hand side of $(5.1)$ evaluates to $(x y \underline{1 / 2}) z \underline{1 / 2}=1 / 2$, while the right-hand side evaluates to

$$
x(y z \underline{\underline{(p \circ q) \rightarrow q)}} \underline{\underline{p \circ q}}=x(y z \underline{\underline{2 / 3}} \underline{\underline{3 / 4}}=1 .
$$

\section{Threshold barycentric algebras}

Definition 6.1. Set a threshold $0 \leq t \leq 1 / 2$. Then the class $\mathcal{B}^{t}$ of threshold- $t$ (barycentric) algebras is the variety generated by the class of convex sets under the threshold-convex combinations of Definition 4.1.

Following Definition 4.1, the operations $\underline{\underline{r}}$ of threshold- $t$ barycentric algebras will be called extreme (small if $0<r<t$, and large if $t^{\prime}<r<1$ ), and moderate if $t \leq r \leq t^{\prime}$.

Remark 6.2. Following Remark 4.2 , the class $\mathcal{B}^{0}$ is just the usual variety $\mathcal{B}$ of barycentric algebras.

If $t>0$, then a threshold- $t$ barycentric algebra $C^{t}=\left(C, \underline{I}^{\circ}\right)$ defined on a convex set $C$ may be considered as an algebra $\left(C,\left[t, t^{\prime}\right],\{\underline{\underline{r}} \mid \underline{r}<t\},\{\underline{\underline{r}} \mid r>\right.$ $\left.t^{\prime}\right\}$ ), where $\left[t, t^{\prime}\right]$ is the set of operations $\underline{\underline{r}}=\underline{r}$ for $t \underline{\overline{\leq r}} \leq t^{\prime}$. In particular, if $t=1 / 2$, then $C^{1 / 2}=(C, \underline{\underline{1 / 2}}=\underline{\underline{1 / 2}},\{\underline{\underline{r}} \mid r<1 / 2\},\{\underline{\underline{r}} \mid r>1 / 2\})$.

Remark 6.3. Threshold-(1/2) convex sets are commutative binary modes under the operation $\star=1 / 2$. As such, they generate the variety $\mathcal{C B M}[16, \mathrm{Ch} 5]$. Note that the elements 0 and 1 of the algebra $(\mathbb{R}, \star)$ generate the unit dyadic interval $\mathbb{D}_{1}$.

In view of Remarks 6.2 and 6.3, it emerges that threshold barycentric algebras offer a spectrum reaching across from barycentric algebras at one end through to commutative binary modes at the other.

The proof of Theorem 5.1 involved an algebra in the class $\mathcal{B}^{1 / 2}$ which is not skew-associative. The following result uses a slightly more refined and general adaptation of that construction.

Theorem 6.4. For a threshold $0 \leq t \leq 1 / 2$, each member of the class $\mathcal{B}^{t}$ of threshold-t barycentric algebras is skew-associative if and only if $t=0$.

Proof. As noted in Remark 6.2, algebras in the class $\mathcal{B}^{0}$ are barycentric algebras. As such, they are certainly skew-associative. 
Now suppose that the threshold $t$ is positive. Consider the closed unit interval $I=[0,1]$ as a convex set. In the skew-associativity identity (5.1), set $p=t$ and $q=1-t$. Then

$$
p \circ q=\left(p^{\prime} q^{\prime}\right)^{\prime}=(q p)^{\prime}=1-t+t^{2}>1-t .
$$

Take $x=1$ and $y=z=0$. Using the inequality (6.1) and the definition (4.1), along with the idempotence of the threshold-convex combinations, the right-hand side of (5.1) evaluates to

$$
1(00 \underline{\underline{(p \circ q) \rightarrow q}}) \underline{\underline{p \circ q}}=00 \underline{\underline{(p \circ q) \rightarrow q}}=0 .
$$

However, the left-hand side evaluates to $(10 \underline{\underline{t}}) 0 \underline{\underline{1-t}}=(1-t) t>0$.

As we have already seen, cancellativity plays an important role in the theory of barycentric algebras and commutative binary modes. The concept is extended to threshold barycentric algebras as follows.

Definition 6.5. Consider a threshold $0 \leq t \leq 1 / 2$, and a threshold- $t$ barycentric algebra $\left(C, \underline{\underline{I}}^{\circ}\right)$. The algebra is cancellative if it satisfies the quasi-identity

$$
(x y \underline{\underline{r}}=x z \underline{\underline{r}}) \rightarrow(y=z)
$$

for all $t \leq r \leq 1-t$. (Compare with (2.5).

Remark 6.6. If a threshold- $t$ barycentric algebra were to satisfy the cancellation quasi-identity (6.2) for any extreme combination $\underline{\underline{r}}$, then it would necessarily be trivial.

The final result of this follows directly by the known properties of barycentric algebras and commutative binary modes (compare Sections 2 and 3.)

Proposition 6.7. Let $\left(C, \underline{\underline{I}}^{\circ}\right)$ be a threshold-t barycentric algebra.

(a) If $t=0$, then $C$ is cancellative if and only if it is a convex set.

(b) If $t=1 / 2$, then $C$ is cancellative if and only if $(C, 1 / 2)$ is a multiplicative subreduct of a quasigroup, or equivalently a $1 / 2$-re $\overline{\overline{d u c t}}$ of an affine space over the ring $\mathbb{D}$.

\section{The variety $\mathcal{B}^{1 / 2}$}

In this section, we show that the varieties $\mathcal{B}^{1 / 2}$ of threshold- $(1 / 2)$ algebras and $\overline{\mathcal{C B M}}$ of extended commutative binary modes are (clonally or term) equivalent. Thus, equivalence is understood in the sense explained in $[16, \S 2.2]$. We conclude the section with some remarks concerning the lattice of subvarieties of the variety $\overline{\mathcal{C B M}}$.

Lemma 7.1. Let $C$ be a convex set. Then the threshold-(1/2) barycentric algebra $\left(C, \underline{\underline{I}}^{\circ}\right)$ and the extended commutative binary mode $(C, \star=\underline{1 / 2}, \triangleleft, \triangleright)$ are equivalènt. 
Proof. Consider the threshold-(1/2) barycentric algebra $C^{1 / 2}$ as an algebra $\left(C, \underline{\underline{I}}^{\circ}\right)=(C, \underline{1 / 2},\{\underline{\underline{r}} \mid r<1 / 2\},\{\underline{\underline{r}} \mid r>1 / 2\})$. Then define the left-zero operation $\triangleleft$ by $x \triangleleft y=x y \underline{r}$ for $r<1 / 2$ and the right-zero operation $\triangleright$ by

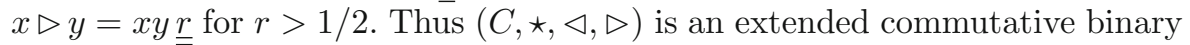
mode. On the other hand, by defining $1 / 2=\star, \underline{\underline{r}}=\triangleleft$ for each $r<1 / 2$, and $\underline{\underline{r}}=\triangleright$ for each $r>1 / 2$ in an extended commutative binary mode, one obtains $\overline{\bar{T}}$ the threshold-(1/2) barycentric algebra $\left(C, \underline{\underline{I}}^{\circ}\right)$.

Lemma 7.1 extends to an equivalence between the corresponding varieties.

Theorem 7.2. The variety $\underline{\underline{B}}^{1 / 2}$ of all threshold-(1/2) barycentric algebras and the variety $\overline{\mathcal{C B M}}$ of extended commutative binary modes are equivalent.

Proof. First recall that the variety $\mathcal{C B M}$ of commutative binary modes is generated by the $1 / 2$-subreducts of dyadic affine spaces, and hence also by $1 / 2$-reducts of real convex sets.

Lemma 7.1 shows that the $\star$-reducts of convex sets, considered as extended commutative binary modes, are equivalent to threshold-(1/2) barycentric algebras. Thus each member of $\mathcal{C B M}$ becomes a threshold-(1/2) barycentric algebra. On the other hand, each threshold-(1/2) barycentric algebra has the structure of an extended commutative binary mode under the operation $\star=\underline{\underline{1 / 2}}$, with $\triangleleft$ equal to any small extreme operation and $\triangleright$ equal to any large extreme operation.

The lattice of varieties of commutative binary modes was first described in [6]. (See also $[14, \S 4.5]$ and $[16, \S 10.4]$.) The lattice of proper subvarieties of $\mathcal{C B M}$ is isomorphic to the product of the two-element lattice and the lattice of odd natural numbers under the divisibility relation. Each proper subvariety of $\mathcal{C B M}$ is defined by one binary identity. The addition of extreme operations $\underline{r}$ for $r \in] 0,1 / 2[\cup] 1 / 2,1[$ to $\mathcal{C B M}$-algebras has no influence on the lattice of subvarieties of these algebras. Thus the lattice of subvarieties of $\mathcal{B}^{1 / 2}$ is isomorphic to the lattice of subvarieties of $\mathcal{C B M}$. Each proper subvariety of $\mathcal{B}^{1 / 2}$ is defined by one binary identity defining the corresponding subvariety of $\mathcal{C B M}$.

\section{Simplices}

Recall that, under the operations of $\underline{I}^{\circ}$, the real $k$-dimensional simplex is the free algebra in $\mathcal{B}$ over the finite set $X=\left\{x_{0}, \ldots, x_{k}\right\}$ of its vertices. It may be characterized as the $\underline{I}^{\circ}$-subreduct

$$
\left\{x_{0} r_{0}+\cdots+x_{k} r_{k} \mid r_{i} \in I, \sum_{i=0}^{k} r_{i}=1\right\}
$$

of the free real affine space $\mathbb{R}^{k}$ over the same set $X$. In particular, the closed unit interval $\left(I, \underline{I}^{\circ}\right)$ is the free barycentric algebra on two free generators 0 and 1. 
The aim of this section is to present Theorem 8.5, showing that for a threshold $0<t<\frac{1}{2}$, each real simplex is generated by threshold-convex combinations of its extreme points.

First recall a fact we will use in the following proofs. For each $p \in I^{\circ}$, and subalgebras $A, B$ of a convex set $\left(C, \underline{I}^{\circ}\right)$, the set $A B \underline{p}:=\{a b \underline{p} \mid a \in A, b \in B\}$ is a subalgebra of $\left(C, \underline{I}^{\circ}\right)$. See $[16, \S 5.1]$, for example.

Lemma 8.1. Let $0<t<\frac{1}{2}$ be a threshold. Consider the closed real unit interval $I$ under the threshold-convex combinations of Definition 4.1. Define

$$
K=\left\lceil\frac{\log t^{\prime}}{\log t-\log t^{\prime}}\right\rceil .
$$

Then the interval $\left[0,\left(t^{\prime}\right)^{K}\right]$ is contained in the subalgebra $A$ of I generated by $\{0,1\}$.

Proof. For a positive integer $h$, one has

$$
t^{h+1}<t^{h} t^{\prime} \leq\left(t^{\prime}\right)^{h} t<\left(t^{\prime}\right)^{h+1}
$$

since $t<t^{\prime}$. Thus

$$
\begin{aligned}
{\left[t^{h+1},\left(t^{\prime}\right)^{h+1}\right] } & =\left[t^{h+1},\left(t^{\prime}\right)^{h} t\right] \cup\left[t^{h} t^{\prime},\left(t^{\prime}\right)^{h+1}\right] \\
& =\left[0 t^{h} \underline{t}, 0\left(t^{\prime}\right)^{h} \underline{t}\right] \cup\left[0 t^{h} \underline{t}^{\prime}, 0\left(t^{\prime}\right)^{h} \underline{t^{\prime}}\right] \\
& =0\left[t^{h},\left(t^{\prime}\right)^{h}\right] \underline{t} \cup 0\left[t^{h},\left(t^{\prime}\right)^{h}\right] \underline{t^{\prime}} .
\end{aligned}
$$

Induction on $h$ then shows that each interval $\left[t^{h},\left(t^{\prime}\right)^{h}\right]$ lies in $A$.

For a positive integer $k$, one has

$$
\begin{gathered}
{\left[t^{k+1},\left(t^{\prime}\right)^{k+1}\right] \cap\left[t^{k},\left(t^{\prime}\right)^{k}\right] \neq \varnothing \Leftrightarrow\left(t^{\prime}\right)^{k+1} \geq t^{k} \Leftrightarrow(k+1) \log t^{\prime} \geq k \log t} \\
\Leftrightarrow 1+\frac{1}{k} \leq \frac{\log t}{\log t^{\prime}} \Leftrightarrow \frac{1}{k} \leq \frac{\log t-\log t^{\prime}}{\log t^{\prime}} \Leftrightarrow k \geq \frac{\log t^{\prime}}{\log t-\log t^{\prime}} .
\end{gathered}
$$

Thus with $K$ as in (8.1), the union

$$
\bigcup_{k=K}^{\infty}\left[t^{k},\left(t^{\prime}\right)^{k}\right]
$$

forms the connected set $\left.] 0,\left(t^{\prime}\right)^{K}\right]$, and the result follows.

Example 8.2. If $t=31 / 64$, then $\left(t^{\prime}\right)^{11}<t^{10}$, but $\left(t^{\prime}\right)^{12}>t^{11}$. Indeed, the quantity

$$
\frac{\log t^{\prime}}{\log t-\log t^{\prime}}
$$

appearing within the right-hand side of (8.1) is approximately 10.595 in this case. Thus $\left[0,\left(\frac{33}{64}\right)^{11}\right]$ lies in $A$.

Remark 8.3. Note that when

$$
0<t \leq \frac{3-\sqrt{5}}{2}
$$

(8.1) yields $K=1$. 
Proposition 8.4. Let $0<t<1 / 2$ be a threshold. Then the closed real unit interval I, under the threshold-convex combinations of Definition 4.1, is generated by $\{0,1\}$.

Proof. With $K$ as in (8.1), set $L=\left(t^{\prime}\right)^{K}$. Consider the open interval $\left.U=\right] 0, L[$. By Lemma 8.1, each element of $U$ may be generated from $\{0,1\}$. By symmetry, each element of $\left.U^{\prime}=\right] 1-L, 1$ [ may be generated from $\{0,1\}$. On the other hand, each element of the dyadic unit interval $\mathbb{D}_{1}=\left\{m 2^{-n} \in I \mid m, n \in \mathbb{N}\right\}$ may also be generated from $\{0,1\}$, merely using the operation $1 / 2$.

Now let $x$ be an arbitrary element of $] \frac{L}{2}, \frac{1}{2}\left[\right.$. Since the set $\mathbb{D}_{1}$ is dense in $I$, there is an element $d$ of $\mathbb{D}_{1}$ with $x-\frac{L}{2}<d<x$. Thus $d<x<d+\frac{L}{2}$. Then

$$
x \in] d, d+\frac{L}{2}\left[=U(2 d) \frac{1}{2}\right.
$$

shows that $x$ may be generated from $\{0,1\}$. By symmetry, each element of ]$\frac{1}{2}, 1-\frac{L}{2}[$ may also be generated from $\{0,1\}$. Since

$$
\left.I \subseteq \mathbb{D}_{1} \cup U \cup U^{\prime} \cup\right] \frac{L}{2}, \frac{1}{2}[\cup] \frac{1}{2}, 1-\frac{L}{2}[,
$$

it follows that the entire interval $I$ is generated by $\{0,1\}$.

Theorem 8.5. Let $0<t<1 / 2$ be a threshold. Then each simplex, under the threshold-convex combinations of Definition 4.1, is generated by its extreme points.

Proof. The proof is by induction on the dimension $n$ of the simplex. For $n=0$, the result is trivial. For $n=1$, it follows by Proposition 8.4. Suppose that the result is true for a positive dimension $n$. Consider the $(n+1)$-dimensional simplex $\Delta_{n+1}$ consisting of all real convex combinations of an $n$-dimensional face $\Delta_{n}$ and an opposing vertex $v$. Thus an arbitrary point $x$ of $\Delta_{n+1}$ lies on a line segment $I$ leading from a point $p$ of $\Delta_{n}$ to $v$. By Proposition 8.4, the point $x$ is generated by threshold-convex combinations of $p$ and the extreme point $v$ of $\Delta_{n+1}$. By induction, the point $p$ is generated by threshold-convex combinations of extreme points of $\Delta_{n}$, which of course are also extreme points of $\Delta_{n+1}$. Thus $x$ is generated by threshold-convex combinations of extreme points of $\Delta_{n+1}$.

\section{The variety $\mathcal{B}^{t}$ with $0<t<1 / 2$}

The aim of this section is to characterize the variety $\mathcal{B}^{t}$ of threshold- $t$ algebras for $0<t<1 / 2$. First note that the extreme operations of threshold- $t$ algebras have no influence on the generation of the algebras considered in Proposition 8.4 and Theorem 8.5. This has an important consequence.

Definition 9.1. Let $\mathcal{B}_{\text {mod }}^{t}$ be the variety generated by the reducts $\left(C, \underline{\left.t, t^{\prime}\right]}\right)$ of threshold- $t$ convex sets with respect to moderate threshold combinations. Members of $\mathcal{B}_{\text {mod }}^{t}$ are called $t$-moderate barycentric algebras. 
Note the following consequence of Theorem 8.5.

Corollary 9.2. Suppose that $0<t<1 / 2$ is a threshold. Then each simplex is generated by its extreme points under the moderate threshold-convex combinations of Definition 4.1.

Proposition 9.3. Suppose that $0<t<1 / 2$ is a threshold. Then each variety $\mathcal{B}_{\text {mod }}^{t}$ of $t$-moderate barycentric algebras is equivalent to the variety $\mathcal{B}$ of usual barycentric algebras.

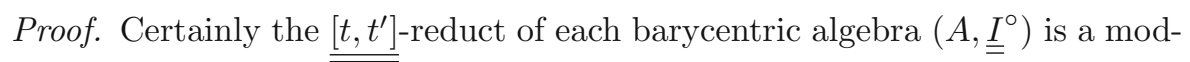
erate barycentric algebra.

Now consider the variety $\mathcal{B}_{\text {mod }}^{t}$ of moderate barycentric algebras for the

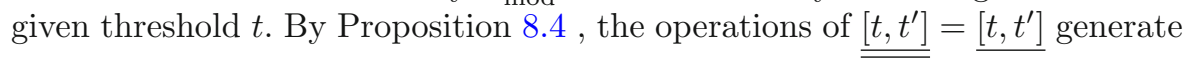
the operations of $\underline{I}^{\circ}$, which are all the binary operations of barycentric algebras. The variety $\mathcal{B}_{\text {mod }}^{t}$ is generated by convex sets. Under the derived operations of $\underline{I}^{\circ}$, they are usual convex sets, satisfy skew-associativity, and generate the variety $\mathcal{B}$ of barycentric algebras.

The following theorem may be proved in similar fashion to Theorem 7.2.

Theorem 9.4. Set a threshold $0<t<1 / 2$. Then each variety $\mathcal{B}^{t}$ of threshold$t$ barycentric algebras is equivalent to the variety $\overline{\mathcal{B}}$ of extended barycentric algebras.

As another consequence of Proposition 8.4 and Theorem 8.5, one obtains the following.

Theorem 9.5. For a threshold $0<t<1 / 2$, the variety $\mathcal{B}^{t}$ is defined by the following identities:

(a) Idempotence, skew-commutativity and entropicity for all operations of $\underline{\underline{I}}^{\circ}$;

(b) The identity $x y \underline{\underline{r}}=x$ for each small operation $\underline{\underline{r}}$;

(c) The identity $x y \underline{\underline{\underline{r}}}=y$ for each large operation $\underline{\underline{\underline{r}}}$;

(d) Skew-associativity for (derived) binary operations generated by the moderate operations $\underline{\underline{r}}$ for $t \leq r \leq t^{\prime}$.

Proof. By the results of Section 4 , members of the variety $\mathcal{B}^{t}$ satisfy the identities of items (a), (b) and (c). By Proposition 8.4 and Theorem 8.5, they satisfy skew-associativity for (derived) binary operations generated by the moderate operations $\underline{\underline{r}}$ for $t \leq r \leq t^{\prime}$. Note that this set of operations coincides with the set of operations $\underline{\underline{r}}$ for $r \in I^{\circ}$.

Now consider algebras $\left(A, \underline{I}^{\circ}\right)$ of the type of $\mathcal{B}^{t}$-algebras satisfying the identities of items (a), (b) and (c), with extreme operations, small for $0<r<t$ and large for $t^{\prime}<r<1$, and skew-associativity for (derived) binary operations generated by the moderate operations $\underline{r}$ for $t \leq r \leq t^{\prime}$. Then under these derived binary operations, members $\left(A, \underline{\underline{\bar{I}}}^{\circ}\right)$ of the variety $V$ lie in the variety of usual barycentric algebras. By Theorem 9.4, the variety $\mathcal{B}^{t}$ may be considered as the variety of usual barycentric algebras additionally equipped with the extreme operations. Hence, the variety $V$, considered as the variety of algebras 
with moderate operations $\underline{r}=\underline{r}$ for $t \leq r \leq t^{\prime}$ and the original extreme operations, is the variety $\mathcal{B}^{\bar{t}}$ of threshold- $t$ barycentric algebras.

The skew-associativity that appears in Theorem 9.5(d) concerns the derived binary operations, but not the basic extreme binary operations. In principle, one could write out the corresponding identities explicitly, with repeated use of the moderate basic operations, but the expressions involved would be very complicated.

\section{Threshold- $t$ semilattices}

Recall that each barycentric algebra has its semilattice replica as a homomorphic image. Moreover the following theorem holds.

Theorem 10.1 [11], [16, Th. 7.6.1]. The variety $\mathcal{B}$ of barycentric algebras contains only one proper non-trivial subvariety, namely the variety $\mathcal{S}$ of (stammered) semilattices.

As each of the varieties $\mathcal{B}^{t}$, for $0<t<1 / 2$, may be considered as the variety of barycentric algebras with additional extreme operations, it is easy to see that each member of such a variety also has a semilattice replica, however equipped additionally with extreme operations of the type of $\mathcal{B}^{t}$-algebras. This leads to the following definition.

Definition 10.2. Let $0 \leq t \leq 1 / 2$ be a threshold. A threshold- $t$ barycentric algebra is a threshold-t semilattice if all moderate operations coincide and are semilattice operations.

Proposition 10.3. Let $0 \leq t \leq 1 / 2$ be a threshold. Then, relative to the variety of threshold-t barycentric algebras, threshold-t semilattices form a subvariety $\mathcal{S}^{t}$ defined by the following identities:

(a) Equality between the respective moderate operations $\underline{p}$, for $t \leq p \leq t^{\prime}$;

(b) Associativity for each moderate operation $\underline{\underline{p}}$, for $t \leq \overline{\bar{p}} \leq t^{\prime}$.

Proof. For $0<t<1 / 2$, idempotence of the moderate operations follows directly by Theorem 9.5(a). Then again by Theorem 9.5(a), one has skewcommutativity for the moderate operations. Given equality of the moderate operations, and the symmetry of the interval $\left[t, t^{\prime}\right]$, their commutativity follows.

For the case $t=1 / 2$, where $1 / 2$ is the only moderate operation, the identities of Proposition 10.3(a) vanish, while Proposition 10.3(b) just specifies the associative law for the commutative binary mode operation $1 / 2$.

By Remark 4.2, the case $t=0$ reduces to the usual situation for extended barycentric algebras, where the equalities (a) suffice, and the associativity (b) then follows from the skew-associativity. 
Remark 10.4. If $t=0$, then the variety $\mathcal{S}^{0}$ is the variety $\mathcal{S}$ of (stammered) semilattices. If $t \neq 0$, then the variety $\mathcal{S}^{t}$ is equivalent to the variety of (stammered) semilattices equipped with additional extreme operations $\underline{\underline{r}}$ for $r<t$ and $r>t^{\prime}$.

As a direct consequence of Theorem 10.1, one obtains the following. Recall (Section 7 ) that the variety $\mathcal{B}^{1 / 2}$ contains infinitely many subvarieties.

Corollary 10.5. Consider a threshold $0 \leq t<1 / 2$. Then the variety $\mathcal{B}^{t}$ of threshold-t barycentric algebras contains only one proper non-trivial subvariety, namely the variety $\mathcal{S}^{t}$ of threshold-t semilattices.

Extended semilattices may be defined in similar fashion to extended barycentric algebras and extended commutative binary modes. In particular, we have the following analogue of Theorems 7.2 and 9.4.

Proposition 10.6. For each positive threshold $t$, the variety $\mathcal{S}^{t}$ of threshold-t semilattices is equivalent to the variety $\overline{\mathcal{S}}$ of extended semilattices.

\section{Barycentric words and identities}

For $0 \leq t \leq 1 / 2$, let $\mathcal{S C}^{t}$ be the variety of algebras of type $I^{\circ} \times\{2\}$, defined by the identities of idempotence, skew-commutativity (2.2) and entropicity (3.1) for all operations of $\underline{\underline{I}}^{\circ}$, along with the left-zero identities for all operations $\underline{p}$ with $0<p<t$ and right-zero identities for all operations $\underline{p}$ with $t^{\prime}<p<\overline{=}$. In particular, for $t=0$, there are no extreme operations, and $\mathcal{S C}^{0}$ coincides with the variety $\mathcal{S C}$ of skew-commutative modes of type $I^{\circ} \times\{2\}$. On the other hand, if $t=1 / 2$, then all the operations $\underline{\underline{p}}$ for $p \neq 1 / 2$ are extreme. Note that the varieties $\mathcal{S C}^{t}$ form a chain, where for $0 \neq t<u$, one has $\mathcal{S C}^{u} \leq \mathcal{S C}^{t}$, and $\mathcal{S C}^{t}<\mathcal{S C}^{0}=\mathcal{S C}$ for all $t \neq 0$. Each variety $\mathcal{B}^{t}$ is a subvariety of $\mathcal{S C}^{t}$. For $t=1 / 2$, the varieties $\mathcal{B}^{1 / 2}$ and $\mathcal{S C}^{1 / 2}$ coincide.

Note also that idempotence and entropicity alone do not define the variety $\mathcal{S C}$. For example, consider an $\underline{\underline{I}}^{\circ}$-algebra $\left(A, \underline{\underline{I}}^{\circ}\right)$, where for each $p \in I^{\circ}$, one has $x y \underline{p}=x$. While the algebra $A$ is idempotent and entropic, it is not skewcommutative.

Proposition 11.1. For each $0 \leq t<1 / 2$, the variety $\mathcal{B}^{t}$ is a proper subvariety of the variety $\mathcal{S C}^{t}$.

Proof. First, note that the variety $\mathcal{B}=\mathcal{B}^{0}$ is a proper subvariety of $\mathcal{S C}$. Now assume that $t \neq 0$. It suffices to provide an example of an algebra which belongs to $\mathcal{S C}^{t}$, but not to $\mathcal{B}^{t}$. Let $A$ be the algebra $\left(I, \underline{\underline{I}}^{\circ}\right)$ defined on the real unit interval $I$ with $\underline{\underline{I}}^{\circ}$-operations defined as follows:

$$
x y \underline{\underline{p}}= \begin{cases}x & \text { if } p<t \\ x y \underline{t} & \text { if } t \leq p<u \\ x y \underline{p} & \text { if } u \leq p \leq u^{\prime} \\ x y \underline{t^{\prime}} & \text { if } u^{\prime}<p \leq t^{\prime} \\ y & \text { if } p>t^{\prime}\end{cases}
$$


for $0<p<1$ and $t<u<1 / 2$. Simple calculations show that the algebra $A$ satisfies the identities defining the variety $\mathcal{S C}^{t}$, but it is not a threshold- $t$ barycentric algebra.

We will now describe free $\mathcal{S C}^{t}$-algebras for $t \neq 0$. Consider a countable set $X=\left\{x_{i} \mid 0<i \in \mathbb{Z}\right\}$ of variables. Let $\mathcal{T}$ denote the set of finite, binary rooted trees. For an element $T \in \mathcal{T}$, let $T_{l}$ denote the set of leaves of $T$. Let $T_{n}$ denote the set of nodes of $T$, including the root. Then let $\lambda_{T}$ or $\lambda: T_{l} \rightarrow X$ and $\nu_{T}$ or $\nu: T_{n} \rightarrow I^{\circ}$ be functions. Each such function pair $\left(\lambda_{T}, \nu_{T}\right)$, for each element $T \in \mathcal{T}$, specifies a word in the language of $\mathcal{S C}$-algebras recursively as follows.

Definition 11.2. Let $T$ be a binary rooted tree.

(a) If $T$ has a unique vertex, then $T_{l}$ is a singleton and $T_{n}$ is empty. If the unique value of $\lambda_{T}$ is $x_{i}$, then that variable is the word specified by the function pair $\left(\lambda_{T}, \nu_{T}\right)$.

(b) Suppose that a rooted binary tree $T$ with root $r$ joins subtrees $T^{\prime}$ and $T^{\prime \prime}$ in order. Then the set $T_{l}$ of leaves of $T$ is the disjoint union of the respective sets $T_{l}^{\prime}$ and $T_{l}^{\prime \prime}$ of leaves of $T^{\prime}$ and $T^{\prime \prime}$, while the set $T_{n}$ of nodes of $T$ is the disjoint union of $T_{n}^{\prime}$ and $T_{n}^{\prime \prime}$ with the singleton $\{r\}$. Suppose that the components of a function pair $\left(\lambda_{T}, \nu_{T}\right)$ restrict to respective function pairs $\left(\lambda_{T^{\prime}}, \nu_{T^{\prime}}\right)$ and $\left(\lambda_{T^{\prime}}, \nu_{T^{\prime}}\right)$, along with a function $\{r\} \rightarrow I^{\circ}$ taking the unique value $p$. Then if the respective barycentric words $u$ and $v$ are specified by $\left(\lambda_{T^{\prime}}, \nu_{T^{\prime}}\right)$ and $\left(\lambda_{T^{\prime}}, \nu_{T^{\prime}}\right)$, the function pair $\left(\lambda_{T}, \nu_{T}\right)$ specifies the word $u v \underline{\underline{p}}$.

A tree $T \in \mathcal{T}$ is complete if each non-leaf node has two children, and all leaves have the same level. The word specified by a complete tree is also called complete. Note that for a complete tree of height $h$, the number of leaves is equal to $l=2^{h}$, and the number of nodes is $n=2^{h+1}-1$.

Let $\mathcal{W}$ be the set of words specified by the trees of $\mathcal{T}$. In a variety $\mathcal{S C}^{t}$, with threshold $0<t \leq 1 / 2$, such words may be reduced to certain special words. First note that by skew-commutativity, for any words $w_{1}$ and $w_{2}$ and any large operation $\underline{p}$, the $\mathcal{S C}^{t}$-algebras satisfy $w_{1} w_{2} \underline{p}=w_{2} w_{1} \underline{p}^{\prime}$, where $\underline{p}^{\prime}$ is small. This allows us to change all appearances of large operations in $w$ into small ones. Next, observe that each small operation $\underline{p}$ satisfies $x y \underline{p}=x$, and more generally $w_{1} w_{2} \underline{\underline{p}}=w_{1}$. This allows one to replace each appearance of subwords of the form $w_{1} w_{2} \underline{\underline{p}}$ in $w$ by $w_{1}$. In this way, one obtains a word $w^{t}$ without symbols of extreme operations, and with all operation symbols belonging to the set $\left[t, t^{\prime}\right]$. Then, using the idempotent laws for all operations of $\left[t, t^{\prime}\right]$, one may extend the word $w^{t}$ to a complete word $w_{c}^{t}$ as follows. If $w^{t}$ contains a subword of the form $u=x v p$, where $v$ is already a complete word specified by a tree $T$ with the function pair $\left(\lambda_{T}, \nu_{T}\right)$, replace $x$ with the word $v_{1}$ specified by the tree $T^{\prime}$ with the function pair $\left(\lambda_{T^{\prime}}, \nu_{T^{\prime}}\right)$ such that $\nu_{T^{\prime}}=\nu_{T}$, and with $\lambda_{T^{\prime}}$ assigning the variable $x$ to each leaf of $T^{\prime}$. The word $w_{c}^{t}$ obtained 
in this way from $w$ is called the canonical form of $w$. Summarizing, one obtains the following proposition.

Proposition 11.3. Set a threshold $0<t \leq 1 / 2$. Then for each word $w \in \mathcal{W}$, the variety $\mathcal{S C}^{t}$ satisfies the identity

$$
w=w_{c}^{t},
$$

where $w_{c}^{t}$ is the canonical form of $w$.

Recall that the word $w_{c}^{t}$ does not contain symbols of extreme operations, but only symbols of operations belonging to $\left[t, t^{\prime}\right]$.

Corollary 11.4. The free $\mathcal{S C}^{t}$-algebra on the set $X$ consists of canonical forms of words in $\mathcal{W}$.

Proof. The proof is by induction on the number $n$ of operational symbols $\underline{p}$

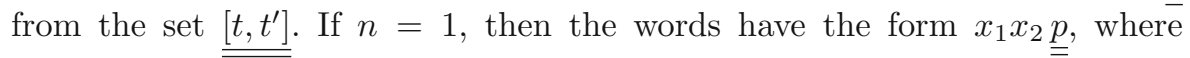
$p \in\left[t, t^{\prime}\right]$ and $\overline{x_{1} \text { and }} x_{2}$ may be equal or different. Hence they are in canonical form. A longer element not in canonical form may be expressed as $w_{1} w_{2} \underline{\underline{p}}$, where $w_{1}$ and $w_{2}$ are canonical words, and $p \in I^{\circ}$. If $\underline{\underline{p}}$ is a small operation, then $w_{1} w_{2} \underline{\underline{p}}=w_{1}$, while if $\underline{\underline{p}}$ is a large operation, then $\overline{\bar{w}}_{1} w_{2} \underline{\underline{p}}=w_{2}$, and hence both are in canonical form. Finally, if $\underline{\underline{p}}$ is a moderate operation, then $w_{1} w_{2} \underline{\underline{p}}$ is in canonical form.

Corollary 11.5. Set a threshold $0<t \leq 1 / 2$. Then for each word $w \in \mathcal{W}$, the variety $\mathcal{B}^{t}$ satisfies the identity

$$
w=w_{c}^{t}
$$

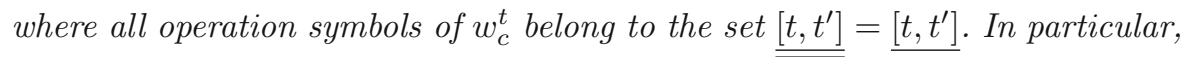
$w_{c}^{t}$ determines an operation of $\mathcal{B}_{\text {mod }}^{t}$-algebras.

\section{Varieties of threshold algebras}

Recall that for a threshold $t$, the symbol $\mathcal{S}^{t}$ denotes the variety of threshold- $t$ semilattices.

Proposition 12.1. For any two thresholds $s$ and $t$ with $0 \leq s<t \leq 1 / 2$, the varieties $\mathcal{S}^{s}$ and $\mathcal{S}^{t}$ are incomparable. Moreover, the meet $\overline{\mathcal{S}^{s}} \wedge \mathcal{S}^{t}$ is the variety $\mathcal{T}$ of trivial algebras.

Proof. First note that if $0 \leq s<t \leq 1 / 2$, then the set of extreme operation symbols of $\mathcal{S}^{t}$-algebras contains the set of extreme operation symbols of $\mathcal{S}^{s}$ algebras. Hence the set of identities defining extreme operations of $\mathcal{S}^{t}$ contains the set of identities defining extreme operations of $\mathcal{S}^{s}$. To show that $\mathcal{S}^{s}$ and $\mathcal{S}^{t}$ are incomparable, consider an operation $\underline{r}$ for $s<r<t$. Then the identity $x y \underline{\underline{r}}=x$ holds in $\mathcal{S}^{t}$, but is not satisfied in $\overline{\mathcal{S}^{s}}$. On the other hand, the variety $\mathcal{S}^{s}$ satisfies the commutative law $x y \underline{\underline{r}}=y x \underline{\underline{r}}$, which is not satisfied in $\mathcal{S}^{t}$. The same laws may be used to show that the meet $\mathcal{S}^{s} \wedge \mathcal{S}^{t}$ is trivial. Indeed, the 
meet $\mathcal{S}^{s} \wedge \mathcal{S}^{t}$ satisfies all identities true in $\mathcal{S}^{s}$ and in $\mathcal{S}^{t}$, and hence $x=x y \underline{\underline{r}}=$ $y x \underline{\underline{r}}=y$.

Corollary 12.2. The varieties $\mathcal{S}^{t}$ of threshold-t semilattices form an antichain. The meet of all $\mathcal{S}^{t}$ is the trivial variety $\mathcal{T}$.

Theorem 12.3. For any two thresholds $s$ and $t$ with $0 \leq s<t \leq 1 / 2$, the varieties $\mathcal{B}^{s}$ and $\mathcal{B}^{t}$ of threshold algebras are incomparable. Moreover, the meet $\mathcal{B}^{s} \wedge \mathcal{B}^{t}$ is the variety $\mathcal{T}$ of trivial algebras.

Proof. If $t \neq 1 / 2$, then it is apparent from Corollary 10.5 and Proposition 12.1 that the varieties $\mathcal{B}^{s}$ and $\mathcal{B}^{t}$ are incomparable and the variety $\mathcal{B}^{s} \wedge \mathcal{B}^{t}$ is trivial.

The only case to consider is $t=1 / 2$. Let $s<t=1 / 2$. Then for each dyadic number $d$ with $s<d<1 / 2$, there is a word $x y w$ in the language of commutative binary modes such that $x y w=x y \underline{d}$. The variety $\mathcal{B}^{s}$ satisfies the identity

$$
x y w=x y \underline{\underline{d}},
$$

but $\mathcal{B}^{1 / 2}$ does not. Consequently, the varieties $\mathcal{B}^{s}$ and $\mathcal{B}^{t}$ are incomparable.

Corollary 12.4. For $t \in[0,1 / 2]$, the varieties $\mathcal{B}^{t}$ of threshold-t barycentric algebras form an antichain. The meet of all $\mathcal{B}^{t}$ is the trivial variety $\mathcal{T}$.

Note that for $t \neq 0$, each variety $\mathcal{B}^{t}$ and its subvariety $\mathcal{S}^{t}$ are strongly irregular. By the theory of regularized varieties (see e.g. [16, §4.3]), it follows that for $t \neq 0$, the regularization $\widetilde{\mathcal{B}^{t}}$ of any variety $\mathcal{B}^{t}$, and similarly the regularization $\widetilde{\mathcal{S}^{t}}$ of any variety $\mathcal{S}^{t}$, consists precisely of Płonka sums of algebras in the original variety, and is defined by the regular identities true in that variety. The regularization is the join of the original variety and the variety $\mathcal{S}$ of semilattices. Note also that in the regularization, the left-zero operations become left-normal operations, and the right-zero operations become rightnormal operations $[16, \S 4.3])$.

In what follows, we will be interested in joins of varieties $\mathcal{B}^{t}$.

Definition 12.5. Set thresholds $0 \leq s<t \leq 1 / 2$. Set $\mathcal{B}^{s, t}$ to be the variety of idempotent, entropic, skew-commutative $\underline{\underline{I}}^{\circ}$-algebras defined by the following identities:

(1) $x y \underline{p}=x$ for all $p<s$;

(2) $x y \underline{\underline{p}}=y$ for all $p>s^{\prime}$;

(3) all identities true in the variety $\mathcal{B}_{\text {mod }}^{t}$ of Definition 9.1 .

The algebra $\left(I, \underline{\underline{I}}^{\circ}\right)$ with appropriately defined extremal operations may be a member of any variety $\mathcal{B}^{t}$. As a member of $\mathcal{B}^{t}$ for $t \neq 0$, it will be denoted by $I^{t}$.

Theorem 12.6. For thresholds $0 \leq s<t \leq 1 / 2$, the join $\mathcal{B}^{s} \vee \mathcal{B}^{t}$ of the varieties $\mathcal{B}^{s}$ and $\mathcal{B}^{t}$ is equal to the variety $\mathcal{B}^{s, t}$. 
Proof. First recall that for any $0<r<1 / 2$, each $\mathcal{B}^{r}$-algebra satisfies the identity $x y \underline{\underline{p}}=x$ for all $p<r$, and all identities true in $\mathcal{B}_{\bmod }^{q}$ for $q \geq r$. Since $s<t$, it follows by Definition 12.5 that any identity true in $\mathcal{B}^{s, t}$ is satisfied in both the varieties $\mathcal{B}^{s}$ and $\mathcal{B}^{t}$, and hence in $\mathcal{B}^{s} \vee \mathcal{B}^{t}$. Consequently, $\mathcal{B}^{s} \vee \mathcal{B}^{t} \leq \mathcal{B}^{s, t}$.

To verify the converse inequality, we will show that each identity true in both $\mathcal{B}^{s}$ and $\mathcal{B}^{t}$ (and hence in $\mathcal{B}^{s} \vee \mathcal{B}^{t}$ ) is also satisfied in $\mathcal{B}^{s, t}$. First note that all left-zero and all right-zero identities true in $\mathcal{B}^{s}$ also hold in $\mathcal{B}^{s} \vee \mathcal{B}^{t}$, and in $\mathcal{B}^{t, s}$.

Now let

$$
w=v
$$

be an identity satisfied in $\mathcal{B}^{s} \vee \mathcal{B}^{t}$ containing operation symbols $p$ for $s \leq p \leq s^{\prime}$. Using skew-commutativity, we may reduce this identity to an identity without large extreme operations. So in what follows, we assume that all the symbols of extreme operations appearing in (12.1) are symbols of left-zero operations.

If all the operation symbols appearing in (12.1) belong to $\underline{\left.\underline{[t}, t^{\prime}\right]}$, then the identity is satisfied by all $\mathcal{B}^{t}$-algebras, and hence by all $\mathcal{B}_{\text {mod }}^{t} \overline{\overline{\text {-algebras. }}}$. Consequently, it holds in all $\mathcal{B}^{s, t}$-algebras. It follows that if the identity (12.1) contains only symbols of left-zero operations $\underline{\underline{p}}$ for all $p<s$, and/or operations from the set $\left[t, t^{\prime}\right]$, then it holds in all $\mathcal{B}^{s, t}$-algebras.

In what follows, assume that (12.1) contains operation symbols $\underline{p}$ only in the range $s \leq p \leq s^{\prime}$. First assume that one side of (12.1), say $w$, contains only operation symbols $p$ for $s \leq p<t$, and the other contains some $p$ with $t \leq p \leq t^{\prime}$. Consider the $\overline{\overline{\mathcal{B}}}{ }^{t}$-algebra $I^{t}$. Substitute 0 and 1 for the variables of (12.1) in any way. Then in $I^{t}, w(0,1)=0$, while $v(0,1)>0$, contradicting the fact that (12.1) holds in $\mathcal{B}^{t}$. Thus if one side contains only $\underline{\underline{p}}$ with $s \leq p<t$, then the same holds also for the other side.

So now assume that both sides of (12.1) contain some operations from $\underline{\left[t, t^{\prime}\right]}$, or all operation symbols of (12.1) are from $\underline{[s, t]}$. By Proposition 9.3, $\overline{\overline{\text { each }}}$ operation $\underline{p}$ for $s \leq p<t$ may be written as a $\overline{\overline{c o m}}$ position of operations $\underline{q}$ for $t<q<t^{\prime}$. Hence the identity may be written as an identity true in all $\overline{\overline{\mathcal{B}}}^{s, t}$-algebras.

Corollary 12.7. Let $0 \leq s, t, u, w \leq 1 / 2$ be thresholds, and let $s<t$ and $u<w$. Then the variety $\mathcal{B}^{s, t}$ is a subvariety of $\mathcal{B}^{u, w}$ if and only if $u \leq s<t \leq w$.

Proof. First assume that $u \leq s<t \leq w$. Note that each $\mathcal{B}^{s, t}$-algebra $A$ satisfies all identities true in $\mathcal{B}_{\text {mod }}^{t}$, and moreover $x y \underline{p}=x$ for all $p<u$. Hence $A$ is a member of $\mathcal{B}^{u, w}$, and consequently $\mathcal{B}^{s, t} \leq \mathcal{B}^{\overline{u, w}}$.

Now let $\mathcal{B}^{s, t} \leq \mathcal{B}^{u, w}$. Assume on the contrary that $u>s$ or $t>w$. First let $u>s$. Note that the $\mathcal{B}^{s}$-algebra $I^{s}$ is a member of $\mathcal{B}^{s, t}$, and hence of $\mathcal{B}^{u, w}$. In particular, each $\mathcal{B}^{s}$-algebra has to satisfy the identity $x y \underline{\underline{p}}=x$ for each $p \in] s, u\left[\right.$. However, in the algebra $I^{s}$, we have $01 \underline{\underline{p}}=01 \underline{p} \neq 0$, a contradiction.

Now assume that $t>w$. Then each $\mathcal{B}^{t}$-algebra, and in particular the algebra $I^{t}$, must be a member of $\mathcal{B}^{u, w}$ and has to satisfy each identity true in 
$\mathcal{B}_{\bmod }^{w}$-algebras. Thus in the $\mathcal{B}^{u, w}$-algebra $I^{t}$, we should have $01 \underline{p}=01 \underline{p}=p$ for each $w<p<t$. However, this is not true, since in the $\mathcal{B}^{t}$-algebra $I^{t}$ we have $01 \underline{\underline{p}}=0$. We again obtain a contradiction. Consequently, $u \leq s<t \leq w$.

Corollary 12.8. The join of the varieties $\mathcal{B}^{s, t}$ is the variety $\mathcal{B}^{0,1 / 2}$, equivalent to the variety $\mathcal{C B M}$ of commutative binary modes.

Proof. Certainly each $\mathcal{B}^{s, t}$, for any $s$ and $t$, is a subvariety of the variety $\mathcal{B}^{0,1 / 2}$. Now the family of all $\mathcal{B}^{s, t}$ contains each of the varieties $B^{0, t}$. Thus the join of all the varieties $\mathcal{B}^{s, t}$ cannot have extremal operations, and since the operation $\underline{1 / 2}$ is the only moderate operation common to all the varieties $\mathcal{B}^{s, t}$, the only $\overline{\overline{m o d}}$ derate operation of the join is $\underline{\underline{1 / 2}}$.

Open Access. This article is distributed under the terms of the Creative Commons Attribution 4.0 International License (http://creativecommons.org/licenses/ by/4.0/), which permits unrestricted use, distribution, and reproduction in any medium, provided you give appropriate credit to the original author(s) and the source, provide a link to the Creative Commons license, and indicate if changes were made.

\section{References}

[1] Davey, B.A., Davis, G.: Tensor products and entropic varieties. Algebra Universalis 21, 68-88 (1985)

[2] Frink, O.: Symmetric and self-distributive systems. Am. Math. Monthly 62, 697$707(1955)$

[3] Gudder, S.: Convex structures and operational quantum mechanics. Comm. Math. Phys. 29, 249-264 (1973)

[4] Ignatov, V.V.: Quasivarieties of convexors, (in Russian). Izv. Vyssh. Uchebn. Zaved. Mat. 29, 12-14 (1985)

[5] Ježek, J., Kepka, T.: Medial Groupoids. Academia, Praha (1983)

[6] Ježek, J., Kepka, T.: The lattice of varieties of commutative abelian distributive groupoids. Algebra Universalis 5, 225-237 (1975)

[7] Loday, J.-L.: Dialgebras. In: Loday, J.L., Frabetti, A., Chapoton, F., Goichot, F. (eds.) Dialgebras and related operads, pp. 7-66. Springer Lecture Notes in Mathematics, Berlin (2001)

[8] Keimel, K.: private communication (2015)

[9] Matczak, K., Romanowska, A.: Quasivarieties of commutative binary modes. Studia Logica 78, 321-335 (2004)

[10] Matczak, K., Smith, J.D.H.: Undirected replicas of directional binary algebras. J. Algebra Appl. 13, 1450051 (2014). (19 pp) 
[11] Neumann, W.D.: On the quasivariety of convex subsets of affine spaces. Arch. Math. (Basel) 21, 11-16 (1970)

[12] Orłowska, E., Romanowska, A.B., Smith, J.D.H.: Abstract barycentric algebras. Fund. Inform. 81, 257-273 (2007)

[13] Ostermann, F., Schmidt, J.: Der baryzentrische Kalkül als axiomatische Grundlage der affinen Geometrie. J. Reine Angew. Math. 224, 44-57 (1966)

[14] Romanowska, A.B., Smith, J.D.H.: Modal Theory. Heldermann, Berlin (1985)

[15] Romanowska, A.B., Smith, J.D.H.: On the structure of barycentric algebras. Houston J. Math. 16, 431-448 (1990)

[16] Romanowska, A.B., Smith, J.D.H.: Modes. World Scientific, Singapore (2002)

[17] Romanowska, A.B., Smith, J.D.H.: Barycentric algebras and gene expression. In: di Gesù, V., Pal, S.K., Petrosino, A. (eds.) WILF 2009, pp. 20-27. Springer Lecture Notes in Artificial Intelligence, Berlin (2009)

[18] Skornyakov, L.A.: Stochastic algebras. Izv. Vyssh. Uchebn. Zaved. Mat. 29, 3-11 (1985)

[19] Smith, J.D.H.: Modes, modals, and barycentric algebras: a brief survey and an additivity theorem. Demonstr. Math. 44, 571-587 (2011)

[20] Smith, J.D.H.: Directional algebras. Houston J. Math. 42, 1-22 (2016)

[21] Smith, J.D.H., Romanowska, A.B.: Post-Modern Algebra. Wiley, New York (1999)

A. Komorowski and A. B. Romanowska

Faculty of Mathematics and Information Sciences

Warsaw University of Technology

00-661 Warsaw

Poland

e-mail [A. Komorowski]: a.komorowski@mini.pw.edu.pl

e-mail [A. B. Romanowska]: aroman@mini.pw.edu.pl

J. D. H. Smith

Department of Mathematics

Iowa State University

Ames IA 50011

USA

e-mail: jdhsmith@iastate.edu

Received: 24 June 2017.

Accepted: 12 December 2017. 